\title{
Role of spironolactone in the treatment of heart failure with preserved ejection fraction
}

\author{
Constantine E. Kosmas ${ }^{1}$, Delia Silverio ${ }^{2}$, Andreas Sourlas ${ }^{3}$, Peter D. Montan ${ }^{2}$, Eliscer Guzman ${ }^{1}$ \\ ${ }^{1}$ Department of Medicine, Montefiore Medical Center, Bronx, NY, USA; ${ }^{2}$ Cardiology Clinic, Cardiology Unlimited, PC, New York, NY, USA; \\ ${ }^{3}$ School of Medicine, University of Crete, Heraklion, Greece \\ Contributions: (I) Conception and design: CE Kosmas; (II) Administrative support: None; (III) Provision of study materials or patients: None; (IV) \\ Collection and assembly of data: All authors; (V) Data analysis and interpretation: All authors; (VI) Manuscript writing: All authors; (VII) Final \\ approval of manuscript: All authors. \\ Correspondence to: Constantine E. Kosmas, MD, PhD. 168-24 Powells Cove Blvd., Beechhurst, NY 11357, USA. Email: cekosmas1@gmail.com.
}

\begin{abstract}
Heart failure (HF) is the leading cause of morbidity and mortality globally. Heart failure with preserved ejection fraction (HFpEF) is currently responsible for about half of the patients affected with $\mathrm{HF}$ and is associated with impaired functional capacity, as well as significant morbidity due to frequent hospitalizations. Unfortunately, despite its poor prognosis, the management of HFpEF is very controversial and no therapy has been so far shown to reduce mortality in HFpEF. Spironolactone antagonizes the effect of aldosterone and can lead to a reduction in fibrosis and an improvement in left ventricular (LV) function. Furthermore, spironolactone decreases extracellular matrix turnover and myocardial collagen content and improves endothelial vasomotor dysfunction, mechanisms known to influence the progression of HF. Thus, given the aforementioned beneficial actions of spironolactone, extensive research has been conducted to explore the effects of spironolactone on HFpEF. Our review aims to present and discuss the clinical and scientific data pertaining to the role of spironolactone in the treatment of patients with HFpEF.
\end{abstract}

Keywords: Spironolactone; aldosterone; renin-angiotensin-aldosterone system (RAAS); heart failure (HF); heart failure with preserved ejection fraction (HFpEF)

Submitted Oct 24, 2018. Accepted for publication Oct 30, 2018.

doi: $10.21037 / \mathrm{atm} .2018 .11 .16$

View this article at: http://dx.doi.org/10.21037/atm.2018.11.16

\section{Introduction}

Heart failure (HF) is the leading cause of morbidity and mortality globally (1). Heart Failure with preserved Ejection Fraction (HFpEF) is currently responsible for about half of the patients affected with HF and is associated with impaired functional capacity, as well as significant morbidity due to frequent hospitalizations (2).

Aldosterone, a mineralocorticoid hormone secreted by the adrenal glands, is considered a causal factor of left ventricular hypertrophy (LVH) by promoting hypertension (HTN) (3). In addition, aldosterone has also been associated with oxidative stress, endothelial dysfunction, myocardial fibrosis and vascular inflammation (4), which are well established factors in the pathogenesis of HF regardless of ejection fraction (EF) $(4,5)$.

Chronic activation of the renin-angiotensin-aldosterone system (RAAS) exerts an important role in the initiation and progression of HF. This chronic activation leads to an increased aldosterone level, which in term stimulates collagen accumulation, resulting in extracellular matrix expansion and endothelial dysfunction (6-8).

Even though the angiotensin converting enzyme inhibitors (ACE-I) and angiotensin II receptor blockers (ARB) suppress angiotensin II-mediated aldosterone release, those drugs do not uniformly suppress the RAAS, resulting in elevated aldosterone levels despite therapy, a phenomenon known as the "aldosterone escape" (9).

Mineralocorticoid antagonists (MRAs), such as spironolactone and eplerenone, have already been shown 
to reduce total and cardiovascular mortality in heart failure with reduced ejection fraction (HFrEF) when administered on top of ACE-I, ARB and beta blockers $(10,11)$.

Spironolactone antagonizes the effect of aldosterone and can lead to a reduction in fibrosis and an improvement in left ventricular (LV) function (6). Furthermore, spironolactone decreases extracellular matrix turnover and myocardial collagen content and improves endothelial vasomotor dysfunction, mechanisms known to affect the progression of HF (12-14).

Thus, given the aforementioned beneficial actions of spironolactone, substantial research has been conducted to explore the effects of spironolactone on HFpEF. Our review aims to present and discuss the clinical and scientific data pertaining to the role of spironolactone in the treatment of patients with HFpEF.

\section{Pathophysiology of HFpEF}

HFpEF, also referred to as diastolic HF, is a clinical entity characterized by signs and symptoms of HF with a normal or low-to-normal ejection fraction. Its incidence and prevalence increase with age (7).

Conventionally, affected individuals present normal LV volume and signs of diastolic dysfunction, such as slowed LV filling and increased filling pressures secondary to increased ventricular stiffness, prolonged isovolumetric $\mathrm{LV}$ relaxation and increased LV end-diastolic pressure $(7,15)$.

Several conditions contribute in the pathogenesis of HFpEF, among them advanced age, HTN, diabetes, coronary artery disease (CAD) and atrial fibrillation, resulting in the development of cardiac structural abnormalities, such as LVH, left atrial dilation and collagen accumulation, leading to extracellular matrix expansion, increased interstitial fibrosis and LV stiffness $(7,16,17)$.

Additionally, dysregulation of titin, a large elastic protein expressed in cardiomyocytes, has been associated with increased cardiomyocyte resting tension or passive stiffness. This protein stores energy during contraction and releases it during relaxation. Changes in the phosphorylation state of titin, or formation of disulfide bridges within the titin molecule, brought about by oxidative stress, have been detected in isolated cardiomyocytes from subjects with HFpEF (17).

\section{Spironolactone in the management of HFpEF}

An important factor in the successful management of
HFpEF is the reduction or the control of the multiple comorbidities associated with this condition, such as HTN, diabetes and atrial fibrillation. In this regard, effective reduction of blood pressure levels decreases LV hypertrophy, reduces LV end-diastolic pressure and improves LV relaxation and filling, thus resulting in a reduction of the progression of HF (18).

MRAs have been of particular interest in the management of HFpEF due to their effects on interstitial fibrosis, myocardial stiffness, extracellular matrix expansion and vascular function, which are all key components in the pathogenesis of HFpEF $(6,19)$.

Spironolactone therapy has been shown to improve the indices of diastolic dysfunction on echocardiography and to decrease procollagen type III $\mathrm{N}$-terminal propeptide accumulation, a circulating marker of myocardial fibrosis (20). Furthermore, there is evidence that spironolactone prevents $\mathrm{LV}$ fibrosis and remodeling after myocardial infarction $(21,22)$.

The Aldo-DHF trial was a multicenter, prospective, randomized, double-blind, placebo-controlled trial, which studied the effect of spironolactone on diastolic function and exercise capacity in 422 patients with HFpEF. The follow-up period of the trial was 12 months and the patients were randomly allocated to receive $25 \mathrm{mg}$ of spironolactone once daily versus matching placebo. Therapy with spironolactone led to an improvement in diastolic function and induced reverse $\mathrm{LV}$ remodeling but did not influence maximal exercise capacity, patient symptoms, or quality of life in patients with HFpEF (23).

In the randomized, double blind Treatment of Preserved Cardiac Function Heart Failure with an Aldosterone Antagonist (TOPCAT) trial, which included 3,445 patients with symptomatic $\mathrm{HF}$ and an $\mathrm{EF} \geq 45 \%$, the patients were randomly allocated to receive $15-45 \mathrm{mg}$ of spironolactone daily versus placebo with a mean followup period of 3.3 years. In this trial, spironolactone failed to produce a significant reduction in the incidence of the primary composite outcome of death from cardiovascular causes, aborted cardiac arrest, or hospitalization for the management of heart failure (24). However, there was a significant correlation between the effect of spironolactone therapy and levels of the natriuretic peptides (NP), with most of the favorable effects of spironolactone observed in subjects with low NP levels, whereas there was no demonstrable benefit in the patients with high NP levels (25).

In a meta-analysis of 14 randomized controlled clinical 
trials, which included 6,428 patients with HFpEF or myocardial infarction with preserved ejection fraction, MRA therapy reduced the number of hospitalizations for HF by $17 \%$, improved diastolic function, induced a reversal of cardiac remodeling and improved quality of life. However, MRA therapy failed to decrease all-cause mortality (26).

In another review of 7 clinical studies evaluating the effects of spironolactone therapy in patients with HFpEF, the incidence of hospitalization for heart failure was significantly lower in the spironolactone group, as compared to placebo. In addition, spironolactone improved diastolic function and caused beneficial remodeling via a reduction in myocardial fibrosis (27).

In the Spironolactone in Myocardial Dysfunction with Reduced Exercise Capacity (STRUCTURE) trial, spironolactone therapy significantly improved the exercise capacity in patients with HFpEF and abnormal diastolic response to exertion. This spironolactone-induced improvement in exercise capacity was mediated by an improvement in the ratio of early mitral inflow velocity to mitral annular early diastolic velocity (E/e') during exercise. Thus, the authors suggested that identification of an elevated $L V$ filling pressure induced by exercise in subjects with HFpEF may select out a subgroup of patients with a higher likelihood of a beneficial response to spironolactone therapy (28).

However, in a large meta-analysis, which included 16,321 patients from 15 randomized controlled trials, MRA therapy led to a decreased risk of cardiovascular death, allcause mortality, and cardiac hospitalizations in subjects with HFrEF but these benefits were not demonstrated in patients with HFpEF. Furthermore, MRA therapy was associated with an increase in the risk of hyperkalemia, whereas treatment with non-selective MRAs was associated with an increase in the incidence of gynecomastia (29).

\section{Conclusions and future directions}

From the above review of the clinical and scientific data, one can conclude that although spironolactone appears to improve diastolic function, induce reverse LV remodeling, and even reduce cardiac hospitalizations and improve quality of life in some studies, on the other hand, there is no definitive demonstrable beneficial effect of spironolactone on all-cause and cardiac mortality in patients with HFpEF. Nonetheless, spironolactone remains as a therapeutic option for HFpEF in patients with evidence of exercise-induced elevation of LV filling pressure, lower NP levels, and structural evidence of diastolic dysfunction with preserved renal function.

Furthermore, as it was alluded before, spironolactone may be more beneficial in certain subgroups of patient with HFpEF. On the other hand, certain other subgroups of patients with HFpEF may be resistant to the favorable effects of spironolactone. In a very recent study, which investigated 381 patients with HFpEF from the Aldo-DHF trial, it was clearly shown that a biochemical phenotype of high collagen cross-linking selects out subjects with HFpEF who are resistant to the favorable effects of spironolactone on LV diastolic dysfunction. The authors postulated that extensive collagen cross-linking, which stabilizes collagen type I fibers, limits the capability of spironolactone to decrease collagen deposition in these patients (30).

Given the above, it becomes evident that further randomized controlled trials are required for a more definitive assessment of the effects of spironolactone in subjects with HFpEF and to potentially identify the subgroups of patients with HFpEF that are most likely to benefit from spironolactone therapy.

\section{Acknowledgements}

None.

\section{Footnote}

Conflicts of Interest: The authors have no conflicts of interest to declare.

\section{References}

1. Benjamin EJ, Blaha MJ, Chiuve SE, et al. Heart Disease and Stroke Statistics-2017 Update: A Report From the American Heart Association. Circulation 2017;135:e146-603.

2. Redfield MM, Jacobsen SJ, Burnett JC Jr, et al. Burden of systolic and diastolic ventricular dysfunction in the community: appreciating the scope of the heart failure epidemic. JAMA 2003;289:194-202.

3. Xanthakis V, Vasan RS. Aldosterone and the risk of hypertension. Curr Hypertens Rep 2013;15:102-7.

4. Briet M, Schiffrin EL. Vascular actions of aldosterone. J Vasc Res 2013;50:89-99.

5. Drazner MH. The progression of hypertensive heart disease. Circulation 2011;123:327-34.

6. Nair A, Deswal A. Aldosterone Receptor Blockade in 
Heart Failure with Preserved Ejection Fraction. Heart Fail Clin 2018;14:525-35.

7. Borlaug BA, Paulus WJ. Heart failure with preserved ejection fraction: pathophysiology, diagnosis, and treatment. Eur Heart J 2011;32:670-9.

8. Edelmann F, Tomaschitz A, Wachter R, et al. Serum aldosterone and its relationship to left ventricular structure and geometry in patients with preserved left ventricular ejection fraction. Eur Heart J 2012;33:203-12.

9. Bomback AS, Klemmer PJ. The incidence and implications of aldosterone breakthrough. Nat Clin Pract Nephrol 2007;3:486-92.

10. Pitt B, Zannad F, Remme WJ, et al. The effect of spironolactone on morbidity and mortality in patients with severe heart failure. Randomized Aldactone Evaluation Study Investigators. N Engl J Med 1999;341:709-17.

11. Zannad F, McMurray JJ, Krum H, et al; EMPHASIS-HF Study Group. Eplerenone in patients with systolic heart failure and mild symptoms. N Engl J Med 2011;364:11-21.

12. Zannad F, Alla F, Dousset B, et al. Limitation of excessive extracellular matrix turnover may contribute to survival benefit of spironolactone therapy in patients with congestive heart failure: insights from the randomized aldactone evaluation study (RALES). Rales Investigators. Circulation 2000;102:2700-6.

13. Bauersachs J, Heck M, Fraccarollo D, et al. Addition of spironolactone to angiotensin-converting enzyme inhibition in heart failure improves endothelial vasomotor dysfunction: role of vascular superoxide anion formation and endothelial nitric oxide synthase expression. J Am Coll Cardiol 2002;39:351-8.

14. Lacolley P, Safar ME, Lucet B, et al. Prevention of aortic and cardiac fibrosis by spironolactone in old normotensive rats. J Am Coll Cardiol 2001;37:662-7.

15. Sharma K, Kass DA. Heart failure with preserved ejection fraction: mechanisms, clinical features, and therapies. Circ Res 2014;115:79-96.

16. Miljkovik LV, Spiroska V. Heart Failure with Preserved Ejection Fraction - Concept, Pathophysiology, Diagnosis and Challenges for Treatment. Open Access Maced J Med Sci 2015;3:521-7.

17. Lewis GA, Schelbert EB, Williams SG, et al. Biological Phenotypes of Heart Failure with Preserved Ejection Fraction. J Am Coll Cardiol 2017;70:2186-200.

18. Velagaleti RS, Gona P, Pencina MJ, et al. Left ventricular hypertrophy patterns and incidence of heart failure with preserved versus reduced ejection fraction. Am J Cardiol 2014;113:117-22.
19. Jia G, Jia Y, Sowers JR. Role of mineralocorticoid receptor activation in cardiac diastolic dysfunction. Biochim Biophys Acta Mol Basis Dis 2017;1863:2012-8.

20. Pandey A, Garg S, Matulevicius SA, et al. Effect of Mineralocorticoid Receptor Antagonists on Cardiac Structure and Function in Patients with Diastolic Dysfunction and Heart Failure with Preserved Ejection Fraction: A Meta-Analysis and Systematic Review. J Am Heart Assoc 2015;4:e002137.

21. Hayashi M, Tsutamoto T, Wada A, et al. Immediate administration of mineralocorticoid receptor antagonist spironolactone prevents post-infarct left ventricular remodeling associated with suppression of a marker of myocardial collagen synthesis in patients with first anterior acute myocardial infarction. Circulation 2003;107:2559-65.

22. Fraccarollo D, Galuppo P, Schmidt I, et al. Additive amelioration of left ventricular remodeling and molecular alterations by combined aldosterone and angiotensin receptor blockade after myocardial infarction. Cardiovasc Res 2005;67:97-105.

23. Edelmann F, Wachter R, Schmidt AG, et al; Aldo-DHF Investigators. Effect of spironolactone on diastolic function and exercise capacity in patients with heart failure with preserved ejection fraction: the Aldo-DHF randomized controlled trial. JAMA 2013;309:781-91.

24. Pitt B, Pfeffer MA, Assmann SF, et al; TOPCAT Investigators. Spironolactone for Heart Failure with Preserved Ejection Fraction. N Engl J Med 2014;370:1383-92.

25. Anand IS, Claggett B, Liu J, et al. Interaction Between Spironolactone and Natriuretic Peptides in Patients With Heart Failure and Preserved Ejection Fraction: From the TOPCAT Trial. JACC Heart Fail 2017;5:241-52.

26. Chen Y, Wang H, Lu Y, et al. Effects of mineralocorticoid receptor antagonists in patients with preserved ejection fraction: a meta-analysis of randomized clinical trials. BMC Med 2015;13:10.

27. Capuano A, Scavone C, Vitale C, et al. Mineralocorticoid receptor antagonists in heart failure with preserved ejection fraction (HFpEF). Int J Cardiol 2015;200:15-9.

28. Kosmala W, Rojek A, Przewlocka-Kosmala M, et al. Effect of Aldosterone Antagonism on Exercise Tolerance in Heart Failure with Preserved Ejection Fraction. J Am Coll Cardiol 2016;68:1823-34.

29. Berbenetz NM, Mrkobrada M. Mineralocorticoid receptor antagonists for heart failure: systematic review and meta- 
analysis. BMC Cardiovasc Disord 2016;16:246.

30. Ravassa S, Trippel T, Bach D, et al. Biomarker-based phenotyping of myocardial fibrosis identifies patients with heart failure with preserved ejection fraction resistant to the beneficial effects of spironolactone: results from the Aldo-DHF trial. Eur J Heart Fail 2018;20:1290-99.

Cite this article as: Kosmas CE, Silverio D, Sourlas A, Montan PD, Guzman E. Role of spironolactone in the treatment of heart failure with preserved ejection fraction. Ann Transl Med 2018;6(23):461. doi: 10.21037/atm.2018.11.16 